\title{
Analysis of passive scalar gradient alignment in a simplified three-dimensional case
}

\author{
A. Garcia and M. Gonzalez \\ CNRS, UMR 6614, Laboratoire de Thermodynamique, CORIA, Site Universitaire du Madrillet, \\ 76801 Saint-Etienne du Rouvray, France
}

(Received 13 January 2006; accepted 22 March 2006; published online 3 May 2006)

\begin{abstract}
Numerical simulations as well as recent experiments in turbulent three-dimensional flow show a trend of the gradient of a passive scalar to align with the compressional axis of the strain tensor. In two-dimensional flow, however, it has been proved that the most probable orientation of the scalar gradient can be different from the compressional direction. An idealized situation is used to address this question in the three-dimensional case and to suggest a possible way to reexamine the scalar gradient alignment in three-dimensional flow. This kind of analysis can be applied to the material line alignment as well. (C) 2006 American Institute of Physics. [DOI: 10.1063/1.2196091]
\end{abstract}

Enhancement of mixing in turbulent flow is closely linked to gradient amplification through small scale production. The mean dissipation rate $\left\langle\epsilon_{\theta}\right\rangle$ of the energy of fluctuations of a scalar $\theta$ is proportional to the variance of the fluctuating gradient through $\left\langle\epsilon_{\theta}\right\rangle=D\left\langle g_{\alpha} g_{\alpha}\right\rangle$, where $D$ is the molecular diffusivity of $\theta$ and $g_{i}=\partial \theta / \partial x_{i}$. The gradient norm and thus dissipation are constantly promoted by strain $s$. This process is expressed by the positivity of the mean production term $-\left\langle g_{\alpha} s_{\alpha \beta} g_{\beta}\right\rangle$ and is, in fact, a kinematic property of the scalar field. ${ }^{1}$

Writing the instantaneous production term in the strain basis reveals that the positivity of its mean value is promoted by alignment of the scalar gradient with the compressional direction $^{1}$

$$
\begin{aligned}
-g_{\alpha} s_{\alpha \beta} g_{\beta}= & -|\boldsymbol{g}|^{2}\left[\lambda_{1} \cos ^{2}\left(\boldsymbol{e}_{1}, \boldsymbol{g}\right)+\lambda_{2} \cos ^{2}\left(\boldsymbol{e}_{2}, \boldsymbol{g}\right)\right. \\
& \left.+\lambda_{3} \cos ^{2}\left(\boldsymbol{e}_{3}, \boldsymbol{g}\right)\right],
\end{aligned}
$$

where the strain eigenvalues are such that $\lambda_{1} \geqslant \lambda_{2} \geqslant \lambda_{3}, \lambda_{1}$ $\geqslant 0, \lambda_{3} \leqslant 0$, and, if incompressibility is assumed, $\lambda_{1}+\lambda_{2}$ $+\lambda_{3}=0$. The strain eigenvectors $\boldsymbol{e}_{1}, \boldsymbol{e}_{2}$, and $\boldsymbol{e}_{3}$ define, respectively, the dilatation, "intermediate," and compression axes of strain. From Eq. (1) and the signs of the $\lambda_{i}$ 's, it is clear that the better the alignment of $\boldsymbol{g}$ with $\boldsymbol{e}_{3}$, the more positive the production term. Alignment properties of the scalar gradient, then, are essential to the mixing process.

Now, in three-dimensional turbulence, numerical simulations $^{1-5}$ as well as recent experimental data ${ }^{6}$ appear to show a trend of the scalar gradient to statistically align with the compressional direction. More precisely, the data only prove that the scalar gradient better aligns with the compression axis than with the other strain directions. This also is a kinematic property. ${ }^{1}$ Note that mean shear, however, seems to weaken the alignment of the gradient with the compressional direction. ${ }^{2,3}$

Even so, the statistical alignment property of the scalar gradient is actually not easy to explain, for the compressional direction is in general not a fixed point of the gradient orientation equations. The compressional direction is the stable fixed point in the special case of a pure, stationary strain, but when vorticity and/or rotation of the strain basis also are present, the existence of an equilibrium orientation for the gradient is generally not proved. ${ }^{7}$ Anyway, the equilibrium orientation, if any, is certainly determined by the combined actions of strain, effective rotation (i.e., vorticity plus strain basis rotation), and molecular diffusion and is not the compressional direction. ${ }^{7}$

This question has already been addressed in twodimensional turbulent flow. ${ }^{8,9}$ Numerical simulations have proved that, in strain-dominated regions, despite a statistical trend of the scalar gradient to align with the compressional direction, the most probable orientation is in fact the equilibrium direction resulting from the competing effects of strain and effective rotation. This direction is known as a simple function of the strain persistence parameter. ${ }^{8}$ Nevertheless, the study of Garcia et al. ${ }^{10}$ suggests that statistical alignment with either the compressional or the equilibrium direction is actually governed by the response of the scalar gradient to Lagrangian fluctuations of strain persistence.

In a three-dimensional flow, the problem is much more complex. As far as we know, even if molecular diffusion is neglected, the equilibrium orientation cannot be derived analytically in the general case and is a priori unknown. There is thus no way (from numerical or experimental data) to check whether or not the scalar gradient statistically aligns with the equilibrium rather than the compressional direction. In this Brief Communication, a simplified three-dimensional case is used to revisit the question of alignment of the scalar gradient with the eigenvectors of the rate-of-strain tensor and to propose a way to examine the preferential orientation of the scalar gradient in a three-dimensional flow.

The flow is assumed to be incompressible. Neglecting molecular diffusion, the Lagrangian equation for the evolution of the scalar gradient is

$$
\frac{d g}{d t}=-s \cdot g+\frac{1}{2} \omega \times g .
$$

From Eq. (2), the equations for the components of $\boldsymbol{g}$ in the strain basis are derived: 


$$
\frac{d g_{i}}{d t}=-\lambda_{i} g_{i}+\frac{1}{2} \varepsilon_{i \alpha \beta}\left(\omega_{\alpha}-\Omega_{\alpha}\right) g_{\beta},
$$

in which $\varepsilon_{i j k}$ is the alternating symbol and $\Omega_{i}$ are the components of the rotation of the strain principal axes, $\Omega_{1}$ $=2 \boldsymbol{e}_{3} \cdot d \boldsymbol{e}_{2} / d t, \Omega_{2}=2 \boldsymbol{e}_{1} \cdot d \boldsymbol{e}_{3} / d t$, and $\Omega_{3}=2 \boldsymbol{e}_{2} \cdot d \boldsymbol{e}_{1} / d t$. In Eq. (3), the summation is taken over the greek indexes.

Expressing $\boldsymbol{g}$ in spherical coordinates in Eq. (3), $\boldsymbol{g}$ $=|\boldsymbol{g}|(\sin \theta \cos \phi, \sin \phi, \cos \theta \cos \phi)$, where $\phi$ is the angle between $\boldsymbol{g}$ and its projection on the plane $\left(\boldsymbol{e}_{1}, \boldsymbol{e}_{3}\right)$ and $\theta$ is the angle between the latter and the compressional direction defined by $\boldsymbol{e}_{3}$, the equations for the orientation of $\boldsymbol{g}$ in the strain basis are derived to be

$$
\begin{aligned}
\frac{d 2 \theta}{d t}= & \left(\lambda_{1}-\lambda_{3}\right)\left(\frac{\omega_{2}^{\prime}-\omega_{1}^{\prime} \tan \phi \sin \theta-\omega_{3}^{\prime} \tan \phi \cos \theta}{\lambda_{1}-\lambda_{3}}\right. \\
& -\sin 2 \theta), \\
\frac{d 2 \phi}{d t}= & \left(\lambda_{1} \sin ^{2} \theta-\lambda_{2}+\lambda_{3} \cos ^{2} \theta\right) \\
& \times\left(\frac{\omega_{3}^{\prime} \sin \theta-\omega_{1}^{\prime} \cos \theta}{\lambda_{1} \sin ^{2} \theta-\lambda_{2}+\lambda_{3} \cos ^{2} \theta}+\sin 2 \phi\right),
\end{aligned}
$$

with $\omega_{i}^{\prime}=\omega_{i}-\Omega_{i}$ and $\phi \neq \pi / 2$. The system of Eqs. (4) and (5) describe the complex behavior of the scalar gradient orientation under the combined actions of strain and effective rotation.

We now assume that vorticity is aligned with a strain eigenvector, namely $\boldsymbol{e}_{2}$; the approach is of course similar (although the results can slightly differ) if $\boldsymbol{\omega}$ is aligned with $\boldsymbol{e}_{1}$ or $\boldsymbol{e}_{3}$ (angles $\theta$ and $\phi$ being appropriately defined). This hypothesis leads to a great simplification, for it is mostly misalignment of vorticity with respect to the strain directions that makes the three-dimensional case difficult. It is, however, relevant insofar as vorticity statistically tends to align with $\boldsymbol{e}_{2},{ }^{2,11,12}$ it is relevant as well in the context of vortex models such as Burgers' and Lundgren's ${ }^{13,14}$ in which vorticity is fully aligned with a strain eigenvector. Although the case under study is idealized, it is a necessary step in attempting to analyze more rigorously the scalar gradient alignment in a three-dimensional flow.

If the fluid is assumed to be inviscid, the $\Omega_{i}$ 's result from two contributions, namely the local action of vorticity expressed by quadratic terms in $\omega_{i} \omega_{j}$ and the nonlocal influence of pressure represented by the off-diagonal components of the pressure Hessian $\boldsymbol{\Pi}$ in the strain basis ${ }^{7}$

$$
\Omega_{i}=-\varepsilon_{\alpha \beta i} \frac{\omega_{\alpha} \omega_{\beta} / 4+\Pi_{\alpha \beta}}{\lambda_{\alpha}-\lambda_{\beta}} .
$$

In a Euler flow, alignment of vorticity with a strain eigenvector is maintained, which implies that rotation of the strain axes is caused by nonlocal effects only. Alignment of vorticity with $\boldsymbol{e}_{2}$ indeed leads to the following: (i) quadratic vorticity terms vanish and (ii) vorticity is also an eigenvector of the pressure Hessian ${ }^{15}$ (which requires the fluid to be inviscid). From (ii), $\boldsymbol{e}_{2}$ is an eigenvector of $\boldsymbol{\Pi}$ and then $\Pi_{12}$
$=\Pi_{23}=0$, which finally leads to $\omega_{1}^{\prime}=\omega_{3}^{\prime}=0$ and $\omega_{2}^{\prime}=\omega_{2}$ $+2 \Pi_{13} /\left(\lambda_{3}-\lambda_{1}\right)$.

Equations (4) and (5) become simplified and, defining $\zeta=2 \theta-\pi / 2$ and normalizing the time by $\lambda_{1}-\lambda_{3}$, they can be written in the form

$$
\begin{aligned}
& \frac{d \zeta}{d \tau}=r_{2}-\cos \zeta, \\
& \frac{d 2 \phi}{d \tau}=\frac{1}{2}\left(\sin \zeta-3 \lambda_{2}^{\star}\right) \sin 2 \phi,
\end{aligned}
$$

in which

$$
\tau=\int_{0}^{t}\left(\lambda_{1}-\lambda_{3}\right) d t^{\prime}, \quad r_{2}=\frac{\omega_{2}^{\prime}}{\lambda_{1}-\lambda_{3}}, \quad \lambda_{2}^{\star}=\frac{\lambda_{2}}{\lambda_{1}-\lambda_{3}} .
$$

Time $t$ is the Lagrangian time. The dimensionless time, $\tau$, is related to the strain-rate history experienced by a fluid particle. Parameter $r_{2}$ defines strain persistence. ${ }^{7-9}$

The analysis does not account for molecular and viscous effects. Previous studies ${ }^{8,9}$ have shown that the former do not significantly influence scalar gradient orientation properties as far as large gradients are considered. The latter are expected not to play a lot provided the Reynolds number is large enough.

Although the flow is not assumed to be locally two dimensional, Eq. (6) is similar to the equation for the scalar gradient orientation in a two-dimensional flow. ${ }^{8,9}$ The twodimensional case is retrieved if $\phi=0$ and $\lambda_{2}=0$ (which implies $\lambda_{1}=-\lambda_{3}$ ).

If $r_{2}^{2}<1$, Eq. (6) has a stable fixed point defined by $\zeta_{\text {eq }}$ $=-\arccos r_{2}$, which (for $\phi=0$ ) corresponds to the compressional direction in the special case $r_{2}=0 .{ }^{8,9}$ Note that $\zeta$ can be constantly close to its equilibrium value even for a timevarying $r_{2}$; this occurs provided the time scale of $r_{2}$ fluctuations is large enough compared to the gradient response time scale, which here is of the order of $\left(\lambda_{1}-\lambda_{3}\right)^{-1} \cdot{ }^{10}$ For $r_{2}^{2}=1$, the equilibrium direction makes an angle of $\pi / 4$ with axes $\boldsymbol{e}_{1}$ and $\boldsymbol{e}_{3}$ whereas for $r_{2}^{2}>1$ rotation prevails and there is no stable orientation. ${ }^{8}$

While an equation similar to Eq. (6) has already been derived in two-dimensional flows, ${ }^{8}$ Eq. (7) is specific to the present three-dimensional case. The value $\phi=0$, corresponding to a scalar gradient oriented in the plane $\left(\boldsymbol{e}_{1}, \boldsymbol{e}_{3}\right)$, is a fixed point of Eq. (7). It is a stable fixed point if $\sin \zeta-3 \lambda_{2}^{\star}$ $<0$. In particular, if $\zeta=\zeta_{\text {eq }}$, then $\phi=0$ is a stable fixed point provided $\left(1-r_{2}^{2}\right)^{1 / 2}+3 \lambda_{2}^{\star}>0$. As a consequence, if strain prevails $\left(r_{2}^{2}<1\right)$ and vorticity is positively stretched $\left(\lambda_{2}>0\right)$, the scalar gradient tends to the plane normal to vorticity, that is, $(\zeta, \phi)$ tends to $\left(\zeta_{\text {eq }}, 0\right)$. If $\lambda_{2}<0$, the scalar gradient does not necessarily tend to be normal to $\boldsymbol{e}_{2}$. Nevertheless, in this case, this trend is promoted by small $r_{2}$ values.

The same method can be used for the material line. In particular, it is found that if $r_{2}^{2}<1$ and $\left(1-r_{2}^{2}\right)^{1 / 2}-3 \lambda_{2}^{\star}>0$, the material line has a stable equilibrium orientation defined by $\zeta_{\mathrm{eq}}^{\text {line }}=-\arccos \left(-r_{2}\right)$ and $\phi=0$. The material line behavior is opposite to that derived for the scalar gradient. If $r_{2}^{2}<1$, the material line tends to its equilibrium orientation in the plane normal to $\boldsymbol{e}_{2}$ for $\lambda_{2}<0$; for $\lambda_{2}>0$, it does not neces- 


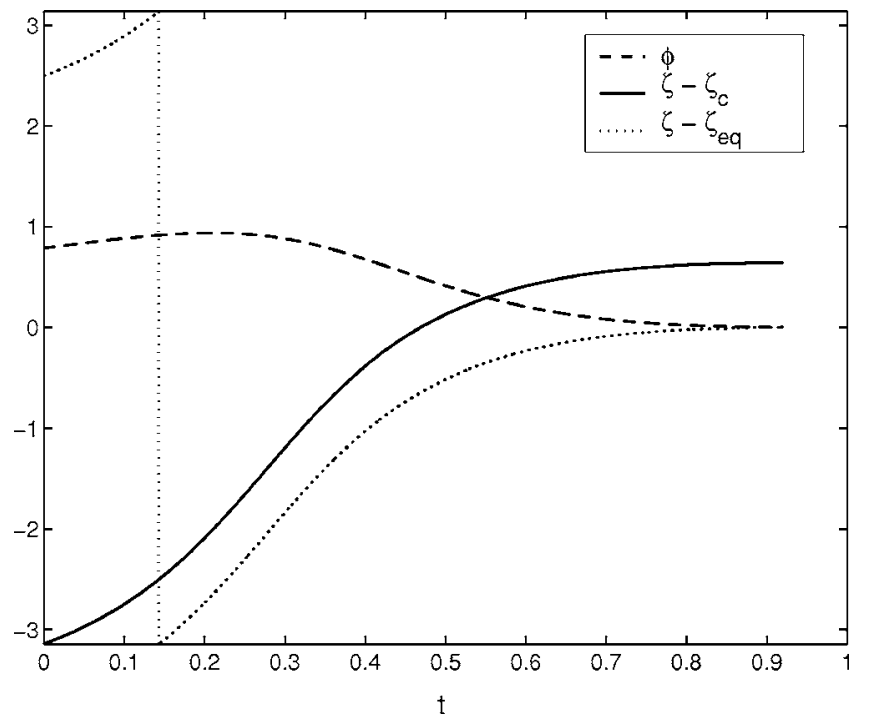

FIG. 1. Evolution of scalar gradient orientation in restricted Euler dynamics for aligned vorticity; initial conditions: $g_{i}(0): 1 / \sqrt{2} ; 1 / \sqrt{2} ; 0 ; \omega_{i}(0): 0 ; 3 ; 0$; $\lambda_{i}(0): 2.4 ; 0.2 ;-2.6$.

sarily tend to be normal to $\boldsymbol{e}_{2}$. The combined effects of strain and effective rotation on material lines alignment were studied by Dresselhaus and Tabor. ${ }^{16}$

If vorticity is assumed to be aligned with $\boldsymbol{e}_{1}$ instead of $\boldsymbol{e}_{2}$, a similar analysis shows that when $r_{1}^{2}<1$ [with $r_{1}$ $\left.=\omega_{1}^{\prime} /\left(\lambda_{2}-\lambda_{3}\right)\right]$, the scalar gradient necessarily tends toward the plane normal to $\boldsymbol{e}_{1}$ because $\left(1-r_{1}^{2}\right)^{1 / 2}+3 \lambda_{1}^{\star}>0$ is always true $\left[\lambda_{1}^{\star}=\lambda_{1} /\left(\lambda_{2}-\lambda_{3}\right)>0\right]$. On the contrary, in this case, the material line never tends to be normal to $\boldsymbol{e}_{1}$ because (1 $\left.-r_{1}^{2}\right)^{1 / 2}-3 \lambda_{1}^{\star}>0$ is never verified (it is easy to show that $\left.3 \lambda_{1}^{\star}>1\right)$.

Different possible trends can be derived from Eqs. (6) and (7) provided $r_{2}$ is constant or at least varies on a time scale larger than the gradient response time scale. The scalar gradient can tend to a stable direction in the plane normal to $\boldsymbol{e}_{2}$ or rotate in the latter; it can also tend to become parallel to $\boldsymbol{e}_{2}$ (when $\phi=0$ is not a stable fixed point) either rotating around the latter or keeping angle $\theta$ constant. In the case where the gradient tends to align with $\boldsymbol{e}_{2}$, however, the asymptotic state is such that either $|\boldsymbol{g}|=0$ (if $\lambda_{2}>0$ ) or $|\boldsymbol{\omega}|$ $=0$ (if $\lambda_{2}<0$ ). These trends have been checked in the simplified case of a "frozen" velocity gradient tensor.

Neglecting the off-diagonal part of the pressure Hessian would lead to a restricted Euler (RE) approximation ${ }^{17}$ of the present approach. With vorticity parallel to a strain eigenvector, the latter implies that there is no rotation of the strain axes and strain persistence thus reduces to $r_{2}=\omega_{2} /\left(\lambda_{1}-\lambda_{3}\right)$. Then, from the RE equations for $\omega_{2}, \lambda_{1}$ and $\lambda_{3}$, ${ }^{18}$ it is straightforward to demonstrate (and thus not reported here) that $r_{2}$ is constant in time. Figure 1 displays the evolution of the scalar gradient toward the stable equilibrium orientation $\left(\zeta_{\text {eq }}=-\arccos 0.6, \phi=0\right)$ (which is different from the compressional direction $\zeta_{c}$ ) in the case where the conditions are such that $r_{2}=0.6$ and $\phi=0$ is a stable fixed point [i.e., (1 $\left.\left.-r_{2}^{2}\right)^{1 / 2}+3 \lambda_{2}^{\star}>0\right]$. These results have been derived by solving the equations for the gradient components together with the RE equations for vorticity and strain eigenvalues. They show that the scalar gradient behavior in RE dynamics is consistent with the present approach.

Finally, in this simplified situation where vorticity is aligned with a strain eigenvector, it appears that the compressional direction is not the equilibrium orientation of the scalar gradient except in the special case of fully persistent strain $\left(r_{2}=0\right)$. There is thus no reason for compression to correspond to the equilibrium orientation in the general case of misaligned vorticity. The common view of a trend to alignment with the compressional direction can therefore be questioned.

Admittedly, the gradient equilibrium orientation must be close to the compressional direction in high strain regions. In the present simple case, the angle between $\boldsymbol{e}_{3}$ and the actual equilibrium direction in the plane $\left(\boldsymbol{e}_{1}, \boldsymbol{e}_{3}\right)$ is smaller than $10^{\circ}$ for $r_{2}<0.35$. Most likely, slight differences between the compressional and equilibrium directions are not observed when plotting, as is usual, the probability density function (pdf) of the cosine of angles instead of the pdf of angles themselves. In regions where strain is moderately large, however, the difference between both directions is not negligible and tends to $\pi / 4$ for $r_{2}$ close to unity. The pdf of $r_{2}$ is therefore crucial; a pdf sharply peaked on zero would imply that the equilibrium orientation is always close to the compressional direction. Nevertheless, numerical simulations of two-dimensional turbulence show that the pdf of strain persistence is rather well distributed around zero. ${ }^{8}$

It is precisely in the two-dimensional case that statistical alignment with the equilibrium orientation depending on local strain persistence has been clearly revealed. ${ }^{8,9}$ Even so, Garcia et al. ${ }^{10}$ have recently shown that the Lagrangian variations of strain persistence $r$ have to occur on a time scale larger than the response time scale of the scalar gradient (which is of the order of $\sigma^{-1}$ where $\sigma$ is the strain intensity) for the latter to be constantly close to the equilibrium orientation defined by the instantaneous $r$. In the opposite case, when strain persistence is fluctuating rapidly with respect to the gradient response, the preferential alignment is determined by $\langle r\rangle$ as $\zeta_{\langle r\rangle}=-\arccos \langle r\rangle$. Then, if $\langle r\rangle \simeq 0$, the preferential orientation turns out to be the compressional direction; this is not because it corresponds to any equilibrium, but just for the reason that the scalar gradient does not keep up with $r$ fluctuations and only feels $\langle r\rangle$.

A similar behavior in the three-dimensional case would imply a possible alignment of the scalar gradient with an equilibrium direction different from the compressional one (at least in strain-dominated regions) if its time response is small enough compared to the time scale of the Lagrangian fluctuations of strain persistence (which, of course, is in general much more difficult to define than in the twodimensional case). This would not be inconsistent with a trend of alignment with the compressional direction, but would just mean the latter is not the most probable one. If, by contrast, the response of the gradient to strain persistence fluctuations is poor, the compressional direction could be the preferential orientation although it is not the equilibrium one.

To summarize, this analysis shows that in threedimensional flow it is still not proved whether or not the compressional direction is the most probable orientation for 
the scalar gradient. This is confirmed, in particular, in the case of the restricted Euler model. A work-in-progress on passive scalar gradient behavior in Burgers' vortex tubes leads to the same conclusion.

Questions remain also regarding the actual mechanism that would impose the compression axis as the preferential orientation. A way of shedding some light on the problem could consist in using three-dimensional, turbulent, Lagrangian data conditioned on persistent alignment of vorticity with a strain eigenvector as recently done by Guala et al. ${ }^{19}$ In the case of strict enough alignment of vorticity, the equilibrium direction could be estimated and the alignments of a scalar gradient with either the latter or the compressional direction could be statistically compared. A similar study could of course be undertaken for the material line by comparing alignment with either the extensional direction or the actual equilibrium orientation.

${ }^{1}$ A. Tsinober and B. Galanti, "Exploratory numerical experiments on the differences between genuine and 'passive' turbulence," Phys. Fluids 15, 3514 (2003).

${ }^{2}$ W. T. Ashurst, A. R. Kerstein, R. M. Kerr, and C. H. Gibson, "Alignment of vorticity and scalar gradient with strain rate in simulated Navier-Stokes turbulence," Phys. Fluids 30, 2343 (1987).

${ }^{3}$ K. K. Nomura and S. E. Elghobashi, "Mixing characteristics of an inhomogeneous scalar in isotropic and homogeneous sheared turbulence," Phys. Fluids A 4, 606 (1992).

${ }^{4}$ J. Martín, C. Dopazo, and L. Valiño, "Joint statistics of the scalar gradient and the velocity gradient in turbulence using linear diffusion models," Phys. Fluids 17, 028101 (2005).

${ }^{5}$ G. Brethouwer, J. C. R. Hunt, and F. T. M. Nieuwstadt, "Micro-structure and Lagrangian statistics of the scalar field with a mean gradient in isotropic turbulence,” J. Fluid Mech. 474, 193 (2003)

${ }^{6}$ B. Galanti, G. Gulitsky, M. Kholmyansky, A. Tsinober, and S. Yorish,
"Joint statistical properties of fine structures of velocity and passive scalar in high Reynolds number flows," in Advances in Turbulence X, Proceedings of the Tenth European Turbulence Conference, edited by H. I. Andersson and P.-Å. Krogstadt (CIMNE, Barcelona, Spain, 2004), pp. 267-270.

${ }^{7}$ M. Tabor and I. Klapper, "Stretching and alignment in chaotic and turbulent flows," Chaos, Solitons Fractals 4, 1031 (1994).

${ }^{8}$ G. Lapeyre, P. Klein, and B. L. Hua, "Does the tracer gradient vector align with the strain eigenvectors in 2D turbulence?" Phys. Fluids 11, 3729 (1999).

${ }^{9}$ G. Lapeyre, B. L. Hua, and P. Klein, "Dynamics of the orientation of active and passive scalars in two-dimensional turbulence," Phys. Fluids 13, 251 (2001).

${ }^{10}$ A. Garcia, M. Gonzalez, and P. Paranthoën, "On the alignment dynamics of a passive scalar gradient in a two-dimensional flow," Phys. Fluids 17, $117102(2005)$

${ }^{11} \mathrm{~K}$. Ohkitani, "Numerical study of comparison of vorticity and passive vectors in turbulence and inviscid flows," Phys. Rev. E 65, 046304 (2002).

${ }^{12}$ B. Lüthi, A. Tsinober, and W. Kinzelbach, "Lagrangian measurements of vorticity dynamics in turbulent flow," J. Fluid Mech. 528, 87 (2005).

${ }^{13} \mathrm{~J}$. M. Burgers, "Application of a model system to illustrate some points of the statistical theory of free turbulence," Proc. R. Acad. Sci. Amsterdam 43, 2 (1940).

${ }^{14}$ T. S. Lundgren, "Strained spiral vortex model for turbulence fine structure," Phys. Fluids 25, 2193 (1982).

${ }^{15} \mathrm{~K}$. Ohkitani, "Eigenvalue problems in three-dimensional Euler flows," Phys. Fluids A 5, 2570 (1993).

${ }^{16} \mathrm{E}$. Dresselhaus and M. Tabor, "The kinematics of stretching and alignment of material elements in general flow fields," J. Fluid Mech. 236, 415 (1991).

${ }^{17} \mathrm{P}$. Vieillefosse, "Internal motion of a small element of fluid in an inviscid flow," Physica A 125, 150 (1984).

${ }^{18}$ K. K. Nomura and G. K. Post, "The structure and dynamics of vorticity and rate of strain in incompressible homogeneous turbulence," J. Fluid Mech. 377, 65 (1998)

${ }^{19}$ M. Guala, B. Lüthi, A. Liberzon, A. Tsinober, and W. Kinzelbach, "On the evolution of material lines and vorticity in homogeneous turbulence," J. Fluid Mech. 533, 339 (2005). 